\title{
Brain Tumor Segmentation with Multi-Path U-Net with Residual Extended Skip Connections
}

This paper was downloaded from TechRxiv (https://www.techrxiv.org).

\section{LICENSE}

CC BY-NC-SA 4.0

SUBMISSION DATE / POSTED DATE

04-07-2021 / 06-07-2021

\section{CITATION}

Sözer, Batuhan; Sözer, Alperen; Şahin, Mustafa Çağlar; Nernekli, Kerem; Erdoğan, Şule Eylem; Çeltikçi, Emrah (2021): Brain Tumor Segmentation with Multi-Path U-Net with Residual Extended Skip Connections. TechRxiv. Preprint. https://doi.org/10.36227/techrxiv.14906724.v1

$\mathrm{DOI}$ 


\title{
Brain Tumor Segmentation with Multi-Path U-Net with Residual Extended Skip Connections
}

\author{
Batuhan Sözer, Alperen Sözer, $M D$, Mustafa Çağlar Şahin, $M D$, Kerem Nernekli $M D$, Şule Eylem \\ Erdoğan, and Emrah Çeltikçi $M D$
}

\begin{abstract}
Early diagnosis of brain tumors is extremely important, and shortening the interval between the acquisition of MRI images and reporting of the results is critical for patients. In the diagnosis of brain tumors, CT and MRI are some of the core diagnostic techniques used today. Our main goal is to reduce the workload of radiologists by developing a neural network that segments MRI images of the brain so we propose a multi-path segmentation algorithm based on U-Net architecture that uses residual extended skip blocks. Our proposed model is trained and tested with Gazi Brains 2020 Dataset. We evaluated the results using the dice similarity coefficient and compared the results with other segmentation algorithms and saw that our proposed model has comparatively better results. Our proposed model is using T1Weighted, T2-Weighted, and Flair MRI images together as inputs, whereas other segmentation models, are using T2-Weighted or Flair MRI images as input. Implementation of the model and trained models are available at https://github.com/batuhansozer/brain-segmentation-with-novelmulti-path-model
\end{abstract}

Index Terms - brain tumor, magnetic resonance imaging, segmentation, deep learning, convolutional neural networks, neuroscience

\section{INTRODUCTION}

Medical decision support systems are gaining popularity as an application field for artificial intelligence. One such research field is image analysis and early detection systems. MRI equipment is becoming more widespread day by day and qualified personnel whom analyses and reports results are overwhelmed by growing piles of non-examined scans. Simultaneously, patients are waiting for MRI results to get a diagnosis and treatment. Sometimes time is crucial for these patients, for example, an aggressive brain tumor, glioblastoma has a median survival of 15 months with maximal treatment (surgery, chemotherapy, and radiotherapy combined) [1]. Any delay to these treatments may result in disease progression and may affect prognosis significantly. A decision support system to pick these tumors from the growing pile of unreported MRI scans and notify the physician would speed up the process for the patient, enhancing prognosis.

Especially with the development of Graphical Processing Unit (GPU) technology and the use of Convolutional Neural Networks (CNNs) gave state-of-art results. While previous researches are being focused on brain tumor detection, in

Author B.S. (e-mail: batuhansozer10@gmail.com) is student at Gazi College, Ankara, TURKEY.

Authors A.S (email: a.sozer.md@gmail.com), M.C.S (email: dr.mcaglarsahin@gmail.com) and E.C (email: drceltikci@gmail.com) is currently affiliated with Gazi University, Faculty of Medicine, Department of Neurosurgery, Ankara, TURKEY. (A.S and M.C.S are neurosurgery residents, E.C is attending neurosurgeon and faculty member) recent years, researchers have been working on semantic segmentation of brain tumors.

Other studies about brain MRI segmentation are mostly done using the BraTS [2] dataset. However, the BraTS dataset is composed of skull-stripped MRI images and only four labels are used to identify structures. Gazi Brains 2020 [3] dataset is used for his study, which contains non-skull-stripped images and 16 different labels are used for segmentation. In realworld applications, algorithms are expected to use nonprocessed MRI data (i.e. non skull-stripped) as input and return results (segmentation, anomaly detection, classification, etc.). A model trained with such minimally processed data is ready to process raw real-world MRI without any additional effort.

In this study, a model that uses T1-Weighted, T2-Weighted, and Flair MRI sequences together as inputs is proposed. Unlike other studies that use only one sequence, this proposed model searches for abnormality in all three sequences. Our model has given better results compared to other models.

\section{RELATED WORK}

U-Net. In recent years after the release of U-Net architecture [4], the segmentation of brain tumors became highly popular. U-Net is a CNN architecture designed for segmenting medical images and now it's one of the most common methods for segmentation tasks. In a study that uses U-Net architecture, Dong et al. [5] proposed an architecture based on U-Net and used BraTS 2015 dataset. They achieved a 0,86 dice coefficient score for whole tumor segmentation with the usage of Flair images.

Residual Network. It has been observed that after a certain depth it became difficult to optimize neural networks to solve the difficulty of optimization in these very deep neural networks He et al. [6] proposed residual extended skip (RES) blocks. They observed a decrease in loss when they introduced RES blocks to existing classification models. After that, adding RES blocks to U-Net architectures gained popularity, Rehman et al. [7] proposed a U-Net based architecture called BU-Net which has residual extended skip (RES) and wide context blocks. They used BraTS 2018 dataset and had a 0.90 dice coefficient score for whole tumor segmentation.

Author K.N (e-mail: kerem.nernekli@gmail.com) was a medical student at Gazi University, Faculty of Medicine, and is not currently affiliated with any instutition.

Author S.E.E (email: sule5001 @yahoo.com) is currently a teacher at Ayranci Anatolian High School. 


\section{MEthoD}

\section{A. Dataset and Preprocessing}

In this study, the Gazi Brains 2020 dataset is used. The dataset contains the MRIs of 100 patients with 50 of them being healthy individuals and 50 of them being patients with GBM. In this dataset, normal anatomical structures, and the abnormal structures -if present- are segmented. The dataset contains T1-Weighted, T2-Weighted, Flair, and post-contrast T1-Weighted MRI (when available) sequences. Post-contrast T1-Weighted MRI has not been used in our study because only patients with GBM and a limited number of normal controls have post-contrast T1Weighted MRI. The dataset also contains segmentation in NIfTi format with 16 different labels.

During the preprocessing phase, MRIs and segmentation masks are resized to $224 \times 224$. In resizing phase, to avoid adding labels that are not meant to be there, Nearestneighbor interpolation [8] is used. The dataset has been split into two parts using a random state of $6,80 \%$ of the dataset is the training set and the remaining $20 \%$ is the test set.

\section{B. Data Augmentation}

Gazi Brains 2020 is a relatively small dataset. In deep neural networks scarcity of samples leads to overfitting, the network memorizes the details rather than generalizing them which leads to failure while making predictions on the test set. To mitigate the overfitting problem caused by the scarcity of samples, data augmentation techniques have been used which is a technique for creating artificial samples. Horizontal and vertical flip, random $90^{\circ}$ rotation, grid distortion, and elastic transform techniques have been used. All the augmentation process is done by using albumentations [9] library with python. Elastic deformation is thought to be very important in this aspect because skull shape may vary between MRI subjects and a portion of these imagings are done after the patient had one or more cranial surgeries. After surgery skull shape may change, changing brain shape and region of interest for the algorithm. Altough our dataset doesn't contain any such image, a portion of the skull is sometimes removed (decompressive craniectomy), significantly distorting brain shape. Training algorithm with such data is an effort to prevent errors caused by this situation.

\section{Proposed Model Architecture}

The proposed model relies on convolution layers, maxpoolig layers, transposed convolution layers, batch normalization [10], residual extended skip blocks [6], ReLU activation function [11], and softmax activation function [12].

Max-pooling layers have been used to downsample the input by half with a pool size of 2 and a stride of 2. Maxpooling layers, in this case, work with a $2 \times 2$ window and takes the maximum value within the window as output. The window is shifted by 2 pixels along each dimension.

Transposed convolution layers have been used to up-sample the image and preferred over up-sampling layers for their kernels that can learn.
Batch normalization has been used after convolution layers and before the activation functions.

Batch normalization is used to make the model more robust to poor initialization. Since neural networks stop improving after it reaches a sufficient depth, residual extended skip blocks have been used to solve that problem. Fig. 1. shows the architecture of the residual extended skip block function used in this architecture.

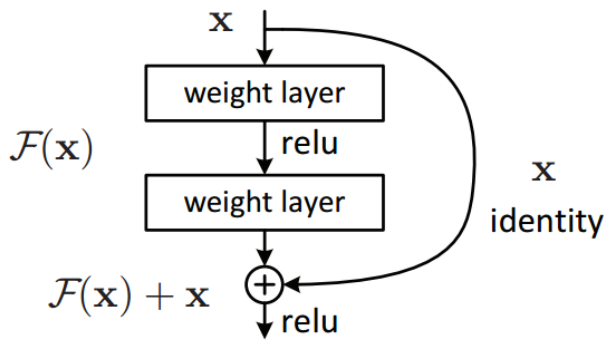

Fig. 1. Residual Extended Skip Block

A momentum of 0.99 and an epsilon of 0.001 is used for batch normalization parameters. Epsilon is a small float value that is added to avoid division by zero.

The ReLU activation function is calculated with the formula of $\operatorname{ReLU}(x)=x^{+}=\max (0, x)$. ReLU activation function solves vanishing gradient problem and it's preferred over sigmoid or tanh function because of the simplicity of computation. ReLU does not include any exponential operations.

The softmax activation function is a generalization of the logistic function to multiple dimensions. The softmax activation function is calculated with the formula of

$$
\sigma(z)_{i}=\frac{e^{z_{i}}}{\sum_{j=1}^{K} e^{z_{j}}} \text { for } i=1, \ldots, K \text { and } z=\left(z_{1}, \ldots, z_{K}\right) \in \mathbb{R}^{K}
$$

Softmax is used in the last layer to normalize the outputs of the convolution layer to a probability distribution over predicted output labels.

The proposed model takes three different inputs, it takes into account T1-Weighted, T2-Weighted, and Flair MRI sequences while making its predictions. The proposed model has three parts which are encoder, bottleneck and decoder. The encoder consists of five residual extended skip blocks and max-pooling layers, it extracts the features of the input and decreases the resolution. The bottleneck consists of one residual extended skip block. And lastly, the decoder consists of five residual extended skip blocks, transposed convolution and merge layers, it takes the feature maps from the encoder via merge layers and increases the resolution of the input back to its original state. The inputs have been passed through from a mutual bottleneck and separate encoders, and decoders. And the resulting feature maps have been concatenated and passed through the final convolutional block with a softmax activation function and 16 filters have been used for this final convolutional layer which is our number of classes. Dropout layers have not been used since no findings of overfitting were observed.

The model scheme can be found in the supplementary materials. 


\section{Training}

Adam optimizer [13] and categorical cross-entropy (CCE) loss function were used. The learning rate is chosen to be 0.0001 , and the batch size was chosen to be 32 . Early stopping with a patience level of 5 was used. The learning rate was reduced with a factor of 0.1 and a patience level of 3 .

$$
C C E=\sum_{i} y_{i} \ln \hat{y}_{i}
$$

where $\mathrm{y}$ is ground-truth value and $\hat{y}$ is prediction value.

In deep neural networks, weight initialization is one of the crucial parts for getting good results [13]. The initial weights should be initialized such that each feature map in the network has the unit variance. While using the ReLU activation function it has proven that He Initialization [14] is a better method than Xavier Initialization [15] by Kumar [16]. He initialization has been used as the weight initialization method since it works better with the ReLU activation function than Xavier Initialization.

\section{E. Implementation}

TensorFlow and Keras [17] is used as backend in the implementation process. All the work has been done with NVIDIA GeForce GTX 2060 graphical processing unit, Intel Core i7-10750H @ 2.60 GHz processor, and 16 GB RAM. Python generators are also used to read the processed data in batches.

\section{RESUlTS AND Discussion}

To compare the performance of the proposed model with other models, two existing models have been implemented which are res u-net which is our baseline model, and multichannel res u-net. For our baseline model the Flair sequence is used as the input and for "multi-channel res u-net" the T2Weighted, T1-Weighted, and Flair sequences are concatenated along the channel axis and used as the input.

Both quantitative and qualitative analyses have been performed. For qualitative analysis, the visual quality of the segmentation has been discussed and for quantitative analysis, the dice coefficient [18] has been used to see the performance of the different models.

$$
D S C=\frac{2 T P}{2 T P+F P+F N}
$$

where TP is true positive values, FP is false positive values, $\mathrm{FN}$ is false negative values.

Since the Gazi Brains 2020 dataset is a non-binary dataset, the dice coefficient has been calculated for each class separately using the evaluate function from the Keras library with a batch size of 32 .
TABLE I

\begin{tabular}{|c|c|c|c|}
\hline $\begin{array}{l}\text { Dice Coefficient } \\
\text { Table }\end{array}$ & $\begin{array}{l}\text { Proposed } \\
\text { Multi-Path } \\
\text { Res U-Net }\end{array}$ & $\begin{array}{l}\text { Baseline } \\
\text { Model }\end{array}$ & $\begin{array}{l}\text { Multi- } \\
\text { Channel } \\
\text { Res U-Net }\end{array}$ \\
\hline Clear Label & 0.98 & 0.98 & 0.98 \\
\hline Region of Interest & 0.94 & 0.94 & 0.93 \\
\hline Edema & 0.62 & 0.60 & 0.57 \\
\hline Lateral Ventricle & 0.74 & 0.72 & 0.61 \\
\hline Eye & 0.76 & 0.82 & 0.72 \\
\hline $\begin{array}{l}\text { Tumor, Contrast } \\
\text { Enhancing Portion }\end{array}$ & 0.54 & 0.55 & 0.43 \\
\hline Tumor, Necrosis & 0.35 & 0.21 & 0.09 \\
\hline Optic Nerve & 0.35 & 0.16 & 0.14 \\
\hline $\begin{array}{l}\text { Tumor, Non- } \\
\text { Contrast } \\
\text { Enhancing Portion }\end{array}$ & 0.27 & 0.12 & 0.16 \\
\hline Third Ventricle & 0.51 & 0.51 & 0.07 \\
\hline $\begin{array}{l}\text { Ischemic Gliotic } \\
\text { Change Areas }\end{array}$ & 0.11 & 0.09 & 0.11 \\
\hline $\begin{array}{l}\text { HaemorrageBlood } \\
\text { (as in Intratumoral } \\
\text { Haemorrage or } \\
\text { else) }\end{array}$ & 0.78 & 0.75 & 0.78 \\
\hline Arachnoid Cyst & 0.57 & 0.57 & 0.57 \\
\hline $\begin{array}{l}\text { Cavum Septum } \\
\text { Pellicidum }\end{array}$ & 0.71 & 0.71 & 0.71 \\
\hline $\begin{array}{l}\text { Late infarct } \\
\text { sequela areas }\end{array}$ & 0.92 & 0.92 & 0.92 \\
\hline Lipoma & 0.92 & 0.92 & 0.92 \\
\hline Mean & 0.63 & 0.60 & 0.54 \\
\hline
\end{tabular}

DICE COEFFICIENT COMPARISON TABLE

The labels are sorted from highest frequency to lowest frequency.

For qualitative analysis, the predictions of all three models have been printed out. A sample from these predictions may be seen in Fig. 2. and all the predictions are made available in our GitHub repository [19].
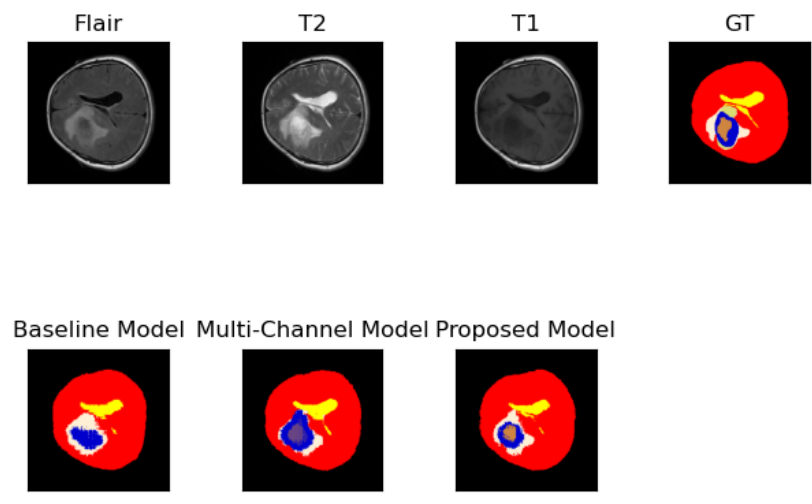

Fig. 2. Sample Prediction for Qualitative Analysis 
To confirm that our model is learning, the graph of the loss function has been drawn using both the training and the validation sets. The loss curves are shown to be similar to each other and both were shown to be decreasing which shows that our model is learning and not overfitting. (Fig. 3.)

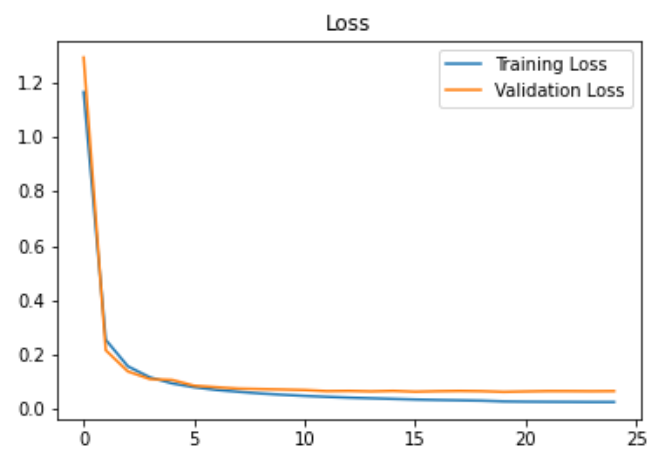

Fig. 3. Loss Curve for Proposed Model

\section{CONCLUSIONS}

MRI segmentation is a difficult task due to the complexity of anatomical structures in the brain. Gazi Brains 2020 dataset contains brain MRIs of $50 \mathrm{GBM}$ patients and brain MRIs of 50 healthy people and normal brain structures are segmented so the class imbalance is reduced.

We propose our novel architecture which takes T1Weighted, T2-Weighted, and Flair MRI sequences together as inputs. Our model approaches the problem like a real radiologist would. We have introduced an architecture that has multiple branches. Each branch has its own encoder, and decoder, and a mutual bottleneck. Each branch extracts features by looking at one sequence at a time and then we use these feature maps to make one final prediction which is our segmentation mask. Our proposed model has been compared with other state-of-art methods and experiments showed that our model has the best performance among them.

Any type of post-processing or usage of loss functions that may improve specific to the dataset is avoided since the main goal is to emphasize the difference between other architectures between our proposed model.

One of the limitations of our study is Gazi Brains 2020 dataset has only 100 patients and one patient has approx. 20 slices each which is not enough to train our model to its full performance, we tried to eliminate this problem using data augmentation techniques. Another limitation of our study is the lack of funding. We tried to use 3D convolution layers and transfer learning techniques but both of these attempts are failed due to our outdated hardware. And we had to reduce our image resolution down to $224 \times 224$ which led to small corruptions both in our MRI data and segmentation masks. More up-to-date systems with higher processing power may eliminate this problem by using higher resolution images and achieve better results. The proposed model can be fine-tuned to reach its full potential.

\section{REFERENCES}

[1] M. S. Greenberg, Handbook of Neurosurgery, 9th edition. Thieme, 2019.

[2] B. H. Menze et al., "The Multimodal Brain Tumor Image Segmentation Benchmark (BRATS)," IEEE Trans Med Imaging, vol. 34, no. 10, pp. 1993-2024, Oct. 2015, doi: 10.1109/TMI.2014.2377694.

[3] S. SAGIROGLU et al., "Gazi Brains 2020 Dataset." Synapse, 2020. doi: $10.7303 / \mathrm{SYN} 22159468$

[4] O. Ronneberger, P. Fischer, and T. Brox, "U-Net: Convolutional Networks for Biomedical Image Segmentation," in Medical Image Computing and Computer-Assisted Intervention-MICCAI 2015, Cham, 2015, pp. 234-241. doi: 10.1007/978-3-319-24574-4_28.

[5] H. Dong, G. Yang, F. Liu, Y. Mo, and Y. Guo, "Automatic Brain Tumor Detection and Segmentation Using U-Net Based Fully Convolutional Networks," Jun. 2017, pp. 506-517. doi: 10.1007/978-3319-60964-5_44.

[6] K. He, X. Zhang, S. Ren, and J. Sun, "Deep Residual Learning for Image Recognition," arXiv:1512.03385 [cs], Dec. 2015, Accessed: Feb. 05, 2021. [Online]. Available: http://arxiv.org/abs/1512.03385

[7] M. U. Rehman, S. Cho, J. H. Kim, and K. T. Chong, "BU-Net: Brain Tumor Segmentation Using Modified U-Net Architecture," Electronics, vol. 9, no. 12, Art. no. 12, Dec. 2020, doi: 10.3390/electronics9122203.

[8] R. Olivier and C. Hanqiang, "Nearest Neighbor Value Interpolation," IJACSA, vol. 3, no. 4, 2012, doi: 10.14569/IJACSA.2012.030405.

[9] A. Buslaev, A. Parinov, E. Khvedchenya, V. I. Iglovikov, and A. A. Kalinin, "Albumentations: fast and flexible image augmentations," Information, vol. 11, no. 2, p. 125, Feb. 2020, doi: 10.3390/info11020125.

[10] S. Ioffe and C. Szegedy, "Batch Normalization: Accelerating Deep Network Training by Reducing Internal Covariate Shift,"p. 9.

[11] X. Glorot, A. Bordes, and Y. Bengio, "Deep Sparse Rectifier Neural Networks," p. 9.

[12] I. Goodfellow, Y. Bengio, and A. Courville, "Deep Feedforward Networks," in Deep Learning, MIT Press, 2016. Accessed: Feb. 07, 2021. [Online]. Available: https://www.deeplearningbook.org/contents/mlp.html

[13] Y. A. LeCun, L. Bottou, G. B. Orr, and K.-R. Müller, "Efficient BackProp," in Neural Networks: Tricks of the Trade, vol. 7700, G. Montavon, G. B. Orr, and K.-R. Müller, Eds. Berlin, Heidelberg: Springer Berlin Heidelberg, 2012, pp. 9-48. doi: 10.1007/978-3-64235289-8_3.

[14] K. He, X. Zhang, S. Ren, and J. Sun, "Delving Deep into Rectifiers: Surpassing Human-Level Performance on ImageNet Classification," in 2015 IEEE International Conference on Computer Vision (ICCV), Dec. 2015, pp. 1026-1034. doi: 10.1109/ICCV.2015.123.

[15] X. Glorot and Y. Bengio, "Understanding the difficulty of training deep feedforward neural networks," in Proceedings of the Thirteenth International Conference on Artificial Intelligence and Statistics, Mar. 2010, pp. 249-256. Accessed: Jun. 04, 2021. [Online]. Available: http://proceedings.mlr.press/v9/glorot10a.html

[16] S. K. Kumar, "On weight initialization in deep neural networks," arXiv:1704.08863 [cs], May 2017, Accessed: Jun. 05, 2021. [Online]. Available: http://arxiv.org/abs/1704.08863

[17] TensorFlow Developers, TensorFlow. Zenodo, 2021. doi: 10.5281/zenodo.4758419.

[18] L. R. Dice, "Measures of the Amount of Ecologic Association Between Species," Ecology, vol. 26, no. 3, pp. 297-302, Jul. 1945, doi: $10.2307 / 1932409$.

[19] B. Sözer, A. Sözer, M. Ç. Şahin, K. Nernekli, Şule Eylem Erdoğan, and E. Çeltikçi, batuhansozer/brain-segmentation-with-novel-multi-pathmodel: Brain Tumor Segmentation with Novel Multi-Path U-Net with Residual Extended Skip Connections. Zenodo, 2021. doi: 10.5281/ZENODO.5009048. 